\title{
Longitudinal rCBF Changes Measured with SPECT in Patients with Depression Undergoing Treatment
}

\author{
Norihiro Miyashita ${ }^{1}$, Hiromichi Ishikawa ${ }^{1}$, Shuntarou Itagaki ${ }^{1}$, Yasuko Takanashi ${ }^{1}$, \\ Takaaki Okano' ${ }^{2}$, Hirobumi Mashiko ${ }^{\text {, Humio Shishido }}{ }^{3}$, Shin-Ichi Niwa ${ }^{4}$ \\ ${ }^{1}$ Fukushima Medical University, School of Medicine, Department of Neuropsychiatry, Fukushima, Japan \\ ${ }^{2}$ Kumamoto Clinic of Psychosomatic Medicine, Kumamoto, Japan \\ ${ }^{3}$ Fukushima Medical University, School of Medicine, Department of Radiology, Fukushima, Japan \\ ${ }^{4}$ Department of Psychiatry, Aizu Medical Center, Fukushima Medical University, Fukushima, Japan \\ Email: nor-miya@ocn.arion.jp
}

How to cite this paper: Miyashita, N., Ishikawa, H., Itagaki, S., Takanashil, Y., Okano, T., Mashiko, H., Shishido, H. and Niwa, S.-I. (2017) Longitudinal rCBF Changes Measured with SPECT in Patients with Depression Undergoing Treatment. Open Journal of Psychiatry, 7, 147-161. https://doi.org/10.4236/ojpsych.2017.73014

Received: April 11, 2017

Accepted: July 4, 2017

Published: July 7, 2017

Copyright ( 2017 by authors and Scientific Research Publishing Inc. This work is licensed under the Creative Commons Attribution International License (CC BY 4.0).

http://creativecommons.org/licenses/by/4.0/

\begin{abstract}
Regional cerebral blood flow (rCBF) studies of major depression have yielded variable results. The present study employed a longitudinal observation method to measure rCBF every 3 months during treatment. Thirteen patients with major depressive disorder underwent single-photon emission computed tomography (SPECT) with ${ }^{99 \mathrm{~m}} \mathrm{Tc}-\mathrm{HMPAO}$ three times over a 6-month period. rCBF was analyzed with the Statistical Parametric Mapping. The findings were compared to scans from 14 normal control subjects. Depression symptoms were rated using the Hamilton Rating Scale for Depression. At baseline, the main regions with lower rCBF compared to controls were the middle and inferior frontal gyri, superior temporal gyrus, and cingulate cortex. Three months later, despite significant improvement of depressive symptoms, decreased $\mathrm{rCBF}$ was observed in the same regions, but to lesser extent. At 6 months, depressive symptoms showed continued improvement, and $\mathrm{rCBF}$ in the superior temporal gyrus increased up to control levels, but $\mathrm{rCBF}$ in the temporal pole, cingulate, and inferior frontal gyrus remained low. The results of the present study suggest that there might be time- and state-dependent differences in $\mathrm{rCBF}$ recovery in patients with major depression.
\end{abstract}

\section{Keywords}

Depression, Regional Cerebral Blood Flow, SPECT, Dorsolateral Prefrontal Cortex, Cingulate Cortex 


\section{Introduction}

Altered brain activity in patients with mood disorders can be reflected in changes in regional cerebral blood flow (rCBF). Many studies have reported rCBF measured by single-photon emission computed tomography (SPECT) in patients with major depression, but comparisons of rCBF with healthy controls and during the course of treatment are inconsistent [1]-[6]. Recovery from depressive symptoms can be obtained in approximately 3 months with antidepressants, but recovery from major depression reportedly requires almost 6 months before the risk of recurrence falls and patient condition stabilizes [7]. The inconsistent results of previous studies may partly be owing to the heterogeneous etiology of depression and personal and treatment conditions. They could also be owing to measurements being taken too early in the course of treatment.

The present study was conducted to solve the problem of inconsistent results in imaging studies. We prospectively followed patients during their treatment course for 6 months and measured rCBF (as a ratio to blood flow in the cerebellum) using SPECT every 3 months. To our knowledge, there have been no reports of serial rCBF measurements performed over 6 months. Our goal was to delineate altered brain function in patients with major depression during exacerbation periods and the alleviation of depressive symptoms following pharmacological treatment.

\section{Subjects and Methods}

\subsection{Subjects}

Subjects were patients with depression who fulfilled the diagnostic criteria for a major depressive episode in the Diagnostic and Statistical Manual of Mental Disorders, $4^{\text {th }}$ edition [8] and whose symptoms were evaluated using the Hamilton Rating Scale of Depression (HRSD, 24-item) [9]. Thirty-two subjects participated in this study. They received pharmaceutical therapy with psychotropic agents, but the types of drugs and their doses were not controlled. Patients suspected of having a serious physical disorder or who had clear abnormalities on magnetic resonance imaging (MRI), X-ray computed tomography (X-CT), electroencephalography (EEG), or an intelligence test (WAIS-R) were excluded. Patients whose HRSD scores did not drop by $30 \%$ or more 3 months after study initiation were also excluded. Thirteen of the $32(40.6 \%)$ subjects had a positive response and participated in the remainder of the study. After 6 months, their HRSD score was less than 10 points. Thirteen patients who met the above criteria were enrolled ( 9 female, 4 male) to complete the study. We also invited healthy control subjects to the present study who had no physical or mental disorders or history of treatment for mental disorders; individuals in this group were not taking any medications. They had no abnormalities on MRI, X-CT, EEG, or intelligence tests. The control group consisted of 14 subjects ( 7 female, 7 male). This study was conducted with the consent of the Fukushima Medical University Ethics Committee (No. 133). Written consent for participation in the 
study was obtained from all subjects.

\subsection{SPECT Imaging}

SPECT was used in rCBF distribution measurements, with ${ }^{99 \mathrm{~m}} \mathrm{Tc}-\mathrm{HMPAO}$ (hexamethylpropyleneamineoxime) $1110 \mathrm{MBq}$ as a radioactive agent given in an intravenous bolus injection via a line secured in the median antebrachial vein. Before and after the injection, the subjects rested in a quiet room with the lights dimmed while wearing an eye mask and ear plugs. Imaging was performed 40 min after injection. Because depressive symptoms such as psychomotor retardation in major depression patients show considerable diurnal variation, ${ }^{99 \mathrm{~m}} \mathrm{Tc}$ HMPAO injection was given between 10 and 11 a.m. SPECT imaging was performed three times, during the depressive phase before treatment (baseline) and 3 and 6 months later. Depressive symptoms were evaluated with the 24-item HRSD within 4 days of each SPECT study.

A Shimadzu Headtome 030 (ring type, HR collimator) was used in SPECT imaging, and image slices were parallel to the orbitomeatal base line (OM line). The obtained images were $64 \times 64$ pixels, and slice thickness was approximately $13 \mathrm{~mm}$.

${ }^{123} \mathrm{I}$ and ${ }^{99 \mathrm{~m}} \mathrm{Tc}$ are the most commonly used isotopes for imaging cerebral blood flow with SPECT. Absolute cerebral blood flow can be calculated by collecting arterial blood when using ${ }^{123} \mathrm{I}$; however, this is not possible when using ${ }^{99 \mathrm{~m}} \mathrm{Tc}$. We calculated $\mathrm{rCBF}$ as a relative value based on the cerebellum as the reference region because cerebellar blood flow is not considered to be affected by factors such as age [10]-[15]. Using the whole brain as the reference region produces inherent contradiction because the target regions are within the reference region.

As a reference site for each imaging, the mean count for the entire cerebellum including the vermis on slices that contained the lower-central pons was calculated, and the value obtained through each pixel count divided by this value was defined as the ratio to the cerebellum used in the analysis.

\subsection{Analysis}

Nine slices were imaged, but the data were rearranged to form 20 slices with resampling (3-dimensional linear method) and expanded (normalized) to a standard brain. They were then compared voxel by voxel using SPM-99 (Statistical Parametric Mapping 99). The significance levels for the intra- and intergroup comparisons were set as $\mathrm{p}<0.005$ and $\mathrm{p}<0.001$, respectively.

Measurements at baseline, 3 months, and 6 months were compared in the subject group using paired $t$-tests $(\mathrm{p}<0.005)$, and between-group comparisons with the healthy control group were performed with two-sample $t$-tests $(\mathrm{p}<$ 0.001). The cluster size threshold was set to greater than 40 voxels for the intraand intergroup comparisons. Image processing was performed with Medex 3.3 (Sensor Systems, Inc.). From this analysis with SPM-99, the anatomical positions of each cluster and Brodmann area were identified using the Talairach atlas [16] 
for locations in which differences or changes were seen. As an indicator of differences between groups on SPM, the Z score ([mean of control group-mean of subject group]/standard deviation of control group) was calculated.

The analyses of other variables such as age, education, and HRSD were conducted using $t$-tests.

\section{Results}

\subsection{Symptoms and Medications}

A total of 13 patients ( 9 female, 4 male) who met the criteria were enrolled. They had a mean age of $46 \pm 13(22-62)$ years and $11.5 \pm 1.7$ years of education. The control group consisted of 14 subjects ( 7 female, 7 male) with a mean age of 31.5 \pm 12.8 years and had $15.4 \pm 3.0$ years of education. The control group was thus significantly younger $(\mathrm{t}(24.31)=2.952, \mathrm{p}=0.007)$ and had significantly more years of education $(t(20.48)=4.099, \mathrm{p}<0.01)$. Depressive symptoms in terms of HRSD scores were $31.5 \pm 12.0$ at baseline, $11.0 \pm 4.3$ at 3 months, and $6.2 \pm 2.7$ at 6 months. The mean score had decreased by $65 \%$ or more at second and third measurements, and these differences were significant (baseline vs. 3 months, $t$ $(12)=6.187, \mathrm{p}<0.01$; baseline vs. 6 months, $\mathrm{t}(12)=8.415, \mathrm{p}<0.01)$. Table 1

Table 1. Daily doses of antidepressant, antipsychotic, and antianxiety medications for each subject at 0,3 , and 6 months.

\begin{tabular}{|c|c|c|c|c|c|c|c|c|c|}
\hline \multirow{2}{*}{ Case no. } & \multicolumn{3}{|c|}{$\begin{array}{l}\text { Imipramine equivalent } \\
\qquad(\mathrm{mg})\end{array}$} & \multicolumn{3}{|c|}{$\begin{array}{l}\text { Chlorpromazine equivalent } \\
\qquad(\mathrm{mg})\end{array}$} & \multicolumn{3}{|c|}{$\begin{array}{c}\text { Diazepam equivalent } \\
(\mathrm{mg})\end{array}$} \\
\hline & $0 \mathrm{~m}$ & $3 \mathrm{~m}$ & $6 \mathrm{~m}$ & $0 \mathrm{~m}$ & $3 \mathrm{~m}$ & $6 \mathrm{~m}$ & $0 \mathrm{~m}$ & $3 \mathrm{~m}$ & $6 \mathrm{~m}$ \\
\hline 1 & 50 & 50 & 50 & 0 & 0 & 0 & 25 & 14 & 14 \\
\hline 2 & 50 & 75 & 75 & 0 & 0 & 0 & 5 & 5 & 5 \\
\hline 3 & 250 & 245 & 150 & 0 & 0 & 0 & 30 & 16 & 8 \\
\hline 4 & 30 & 30 & 30 & 0 & 0 & 0 & 10 & 15 & 15 \\
\hline 5 & 90 & 33 & 33 & 110 & 70 & 70 & 5 & 13 & 8 \\
\hline 6 & 125 & 225 & 225 & 50 & 60 & 138 & 11 & 11 & 7 \\
\hline 7 & 10 & 10 & 10 & 15 & 30 & 50 & 6 & 6 & 6 \\
\hline 8 & 10 & 15 & 15 & 0 & 26 & 300 & 5 & 17 & 2 \\
\hline 9 & 40 & 150 & 125 & 0 & 130 & 160 & 4 & 4 & 4 \\
\hline 10 & 50 & 100 & 75 & 0 & 0 & 0 & 18 & 10 & 10 \\
\hline 11 & 45 & 65 & 45 & 0 & 0 & 0 & 0 & 0 & 0 \\
\hline 12 & 0 & 0 & 0 & 50 & 0 & 0 & 3 & 2 & 2 \\
\hline 13 & 13 & 125 & 125 & 0 & 0 & 0 & 5 & 5 & 5 \\
\hline Avg & 59.4 & 89.4 & 75.7 & 18.7 & 26.3 & 59.8 & 8.5 & 8.7 & 6 \\
\hline Std & 69.9 & 82.6 & 68.3 & 34.5 & 41.2 & 94.9 & 8.2 & 5.8 & 4 \\
\hline
\end{tabular}

a. The doses (mg/day) of antidepressants, antipsychotics, and antianxiety medications are expressed in imipramine, chlorpromazine, and diazepam equivalents, respectively. Equivalents were calculated based on the conversion table formulated by the clinical psycho-pharmacology study group of Keio University [17]. Avg, average; Std, standard deviation. 
shows the antidepressant, antipsychotic, and antianxiety drug amounts in equivalent dosages of imipramine, chlorpromazine, and diazepam, respectively, that were taken by patients at each time point. The conversion in equivalent dosages of imipramine, chlorpromazine, and diazepam was calculated based upon the conversion table formulated by clinical psycho-pharmacology study group of Keio University [17]. The mean medication doses per day, in imipramine, chlorpromazine, and diazepam equivalents, were not significantly different between the three time points.

\section{2. rCBF Distribution}

\subsubsection{Comparison of Patients with Healthy Controls}

The regions where rCBF differences were seen in patients compared to the control group are listed in Table 2. At baseline, areas with lower rCBF ratios to the cerebellar blood flow as compared to the control subjects were the bilateral superior temporal gyri (temporal pole including amygdala), bilateral cingulate (frontal portion), right inferior frontal gyrus, bilateral middle frontal gyri, left anterior cingulate (antero-inferior), and left middle and superior frontal gyri.

The lower rCBF areas improved at 3 and 6 months, particularly in the lateral prefrontal cortices and cingulate gyrus. After 3 months, there were no longer differences in $\mathrm{rCBF}$ between the two groups in the right inferior frontal gyrus (orbitofrontal area), left middle frontal gyrus, left anterior cingulate (antero-inferior), or left middle and superior frontal gyri (Table 2).

After 6 months, the lower rCBF areas of patients with depression were no longer significant compared to controls in the right inferior frontal gyrus (orbitofrontal area), right superior temporal gyrus (temporal pole), bilateral middle frontal gyri, anterior cingulate (antero-inferior), and middle and superior frontal gyri (Table 2, Figure 1). There were no areas with higher rCBF in the patient group compared to the control group at baseline. After 3 months, patients had higher rCBF than controls in the bilateral superior temporal gyrus, bilateral inferior temporal gyri, left middle temporal gyrus, left middle occipital gyrus, right lingual gyrus, bilateral fusiform gyri, and right parahippocampal gyrus. These higher $\mathrm{rCBF}$ areas were also recognized at 6 months (except the right parahippocampal gyrus). Instead, the middle temporal gyrus of the patients showed higher rCBF than the controls de novo (Figure 2).

As there was a significant difference in mean age between the patient and control groups, we attempted to recruit an additional control group whose age corresponded to those of the patients; however, the Headtome $\gamma$-camera used in this study was unavailable during new data collection due to unforeseen circumstances. Therefore, we were unable to gather data from this older control group. To compensate the failed attempt to add control subjects, we examined the effect of age on $\mathrm{rCBF}$ in five representative regions of interest (ROIs: cingulate, caudate, putamen, thalamus, and dorsolateral prefrontal cortex [DLPFC]) in patients at baseline by assessing the correlation between age and bilateral rCBF. The Pearson correlation coefficient was $0.068(\mathrm{p}=0.442)$, indicating no correlation between age and $\mathrm{rCBF}$ in our patient group. 
Table 2. Comparison between the depression and control groups.

\begin{tabular}{|c|c|c|c|c|}
\hline Comparison & Brain region & Left or right & Brodmann area & Z score \\
\hline \multirow{9}{*}{0 months $<$ control } & Middle \& superior frontal gyri & Left & 10 & 4.68 \\
\hline & Middle frontal gyrus & Right & 10,46 & 4.57 \\
\hline & Middle frontal gyrus & Left & 11 & 5.02 \\
\hline & Inferior frontal gyrus (orbitofrontal area) & Right & 47 & 5.02 \\
\hline & Inferior frontal gyrus & Right & 44 & 4.70 \\
\hline & Cingulate (frontal portion) & Both & 32 & 5.10 \\
\hline & Cingulate (antero-inferior) & Left & 32 & 4.99 \\
\hline & Superior temporal gyrus (temporal pole) & Left & 38 & 5.79 \\
\hline & Superior temporal gyrus (temporal pole) & Right & 38 & 4.42 \\
\hline \multirow{5}{*}{3 months $<$ control } & Middle frontal gyrus & Right & 10,9 & 4.21 \\
\hline & Inferior frontal gyrus & Right & 47 & 4.6 \\
\hline & Cingulate (frontal portion) & Both & 32 & 4.14 \\
\hline & Superior temporal gyrus (temporal pole) & Left & 38 & 5.31 \\
\hline & Superior temporal gyrus (temporal pole) & Right & 38 & 4.25 \\
\hline \multirow{3}{*}{6 months $<$ control } & Inferior frontal gyrus & Right & 47 & 4.22 \\
\hline & Cingulate (frontal portion) & Both & 32 & 4.33 \\
\hline & Superior temporal gyrus (temporal pole) & Left & 38 & 5.48 \\
\hline 0 months $>$ control & n. d. & & & \\
\hline \multirow{8}{*}{3 months $>$ control } & Parahippocampal gyrus & Right & 27 & 3.95 \\
\hline & Middle temporal gyrus & Left & 21 & 4.46 \\
\hline & Superior temporal gyrus & Both & 22 & 5.04 \\
\hline & Inferior temporal gyrus & Right & 37 & 3.92 \\
\hline & Fusiform gyrus & Both & 37 & 4.37 \\
\hline & Middle occipital gyrus & Left & 19,37 & 4.44 \\
\hline & Lingual gyrus & Right & 18 & 4.37 \\
\hline & Cuneus & Right & 17,18 & 3.75 \\
\hline \multirow{6}{*}{6 months $>$ control } & Middle temporal gyrus & Right & 21 & 4.92 \\
\hline & Superior temporal gyrus & Left & 22 & 4.14 \\
\hline & Fusiform gyrus & Both & 37 & 4.12 \\
\hline & Middle occipital gyrus & Both & 19 & 5.18 \\
\hline & Lingual gyrus & Both & 18 & 4.60 \\
\hline & Cuneus & Both & 17,18 & 4.60 \\
\hline
\end{tabular}

a. The regions with significant differences in blood flow between the depression and control groups for 0 (baseline), 3 , and 6 months after treatment initiation are listed. $\mathrm{Z}$ scores represent the biggest differences in blood flow across voxels within the region. n.d.: not detected. 

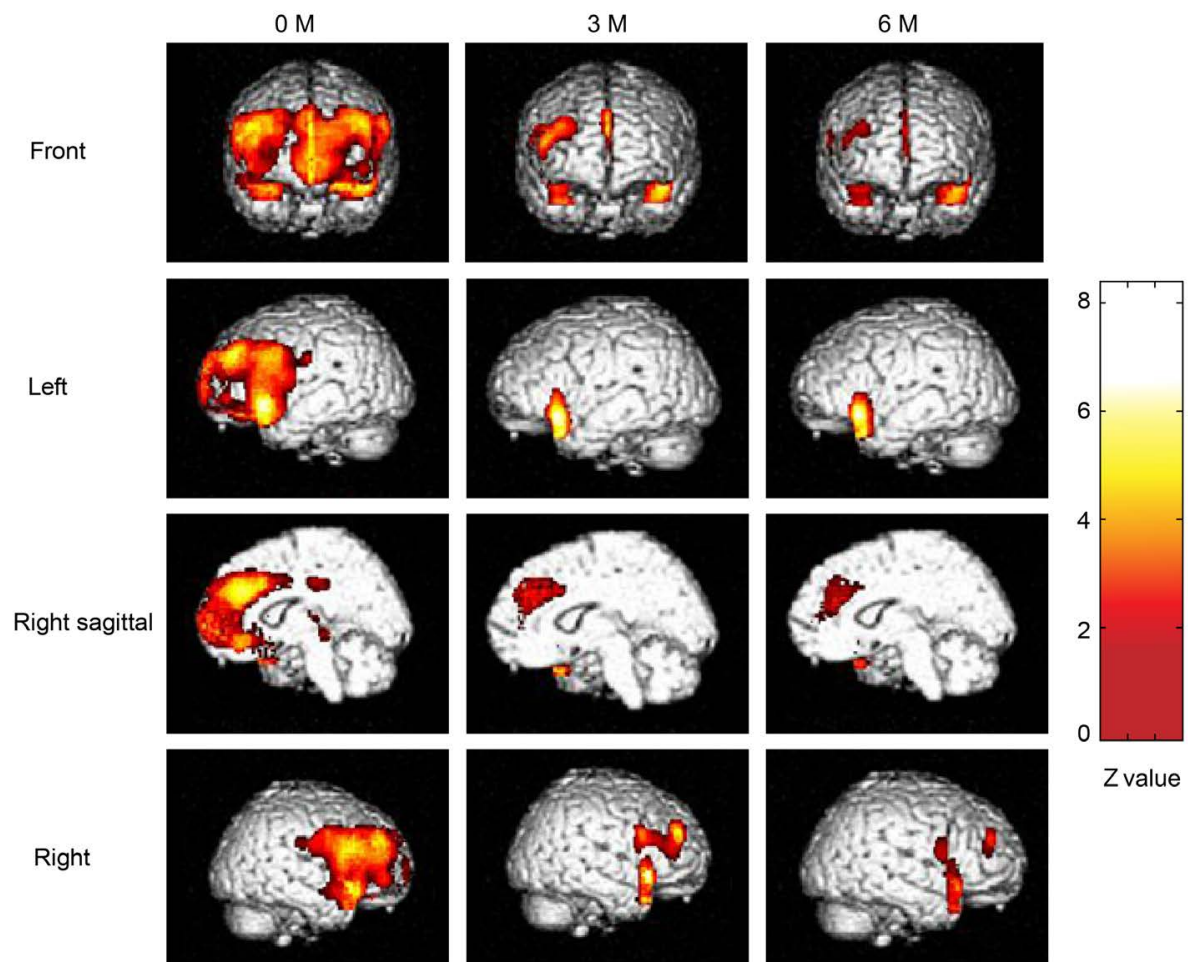

Z value

Figure 1. Brain regions in which patients' blood flow was lower than that of controls. The colored areas represent regions with significantly lower blood flow ( $t \geq 3.725$ with 25 degrees of freedom, $\mathrm{p} \leq 0.0001)$.

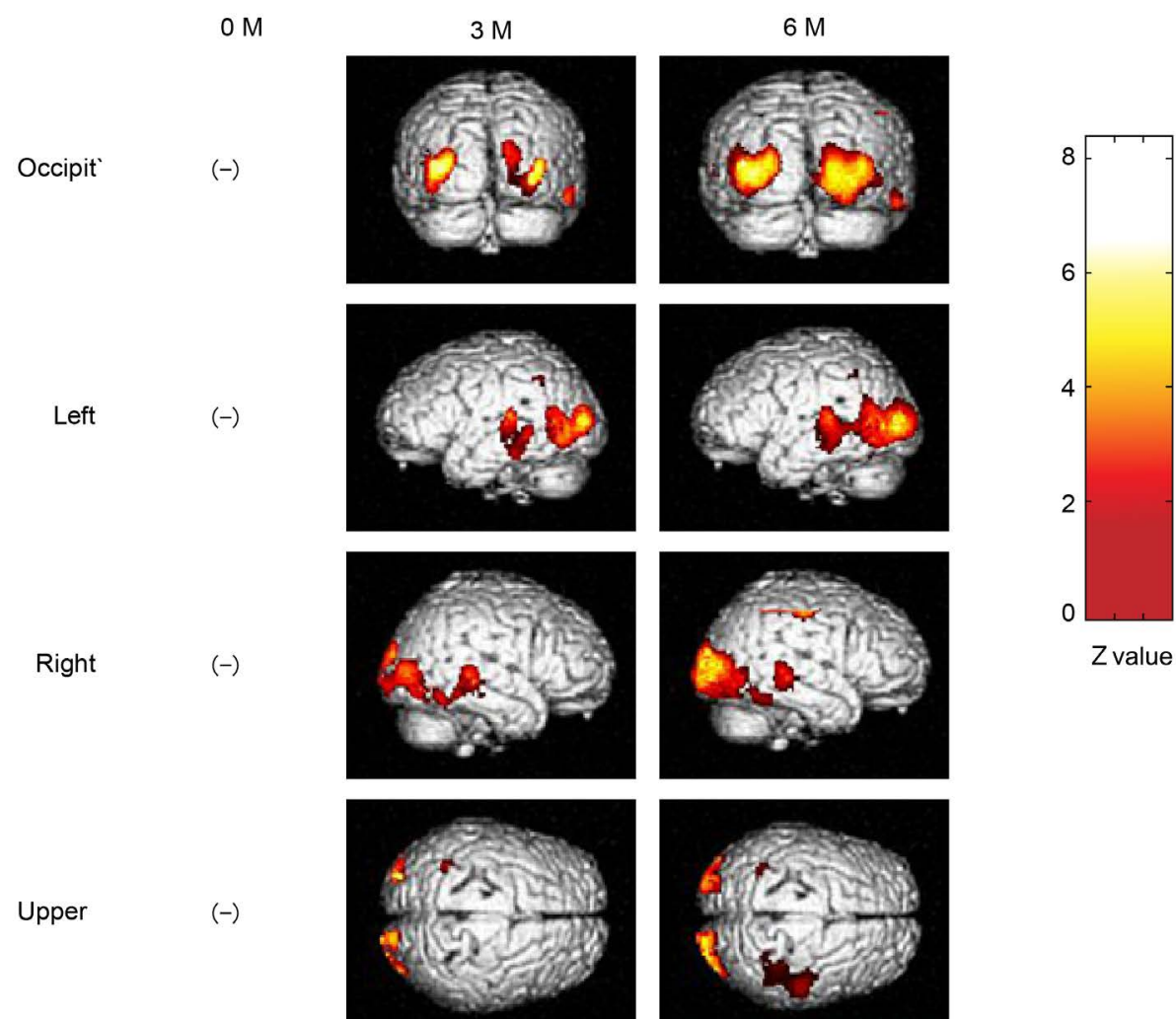

Figure 2. Brain regions in which patients' blood flow was higher than that of controls. The colored areas represent regions with significantly higher blood flow ( $t \geq 3.725$ with 25 degrees of freedom, $\mathrm{p} \leq 0.0001$ ). 


\subsubsection{Comparison within the Subject Group}

Anatomical locations and Brodmann areas that changed over time among the subjects in the depression group are shown in Table 3. The areas in which rCBF increased from baseline to 3 months were the left superior frontal gyrus, left middle frontal gyrus, and left precentral gyrus, and middle temporal gyrus, and those in which $\mathrm{rCBF}$ decreased over that time were the right orbitofrontal gyrus, right superior temporal gyrus, right precentral gyrus, right sub-lobar insula, and left superior temporal gyrus. The areas in which rCBF increased from months 3 to 6 were the left anterior cingulate gyrus, left precuneus, left cuneus, left lingual and fusiform gyri, right superior frontal gyrus, and right middle frontal gyrus. Those in which rCBF decreased from months 3 to 6 were the left inferior frontal gyrus, left temporal tip, left middle temporal gyrus, and right superior temporal gyrus (Table 3).

Table 3. Comparison within the depression group.

\begin{tabular}{|c|c|c|c|c|}
\hline Comparison & Brain region & Left or right & Brodmann area & Z score \\
\hline \multirow{5}{*}{3 months $>0$ months } & Middle frontal gyrus & Left & 10 & 3.30 \\
\hline & Superior frontal gyrus & Left & 9 & 2.75 \\
\hline & & & & \\
\hline & Middle temporal gyrus & Left & 21 & 3.21 \\
\hline & Precentral gyrus & Left & 4 & 3.82 \\
\hline \multirow{6}{*}{3 months $<0$ months } & Orbitofrontal gyrus & Right & 11 & 3.16 \\
\hline & Sub-lobar insula & Right & 13 & 3.46 \\
\hline & Superior temporal gyrus (mid portion) & Right & 22 & 3.89 \\
\hline & Superior temporal gyrus (posterior) & Left & 22 & 3.40 \\
\hline & Superior temporal gyrus (anterior) & Right & 22 & 3.26 \\
\hline & Precentral gyrus & Right & 4 & 3.19 \\
\hline \multirow{7}{*}{6 months $>3$ months } & Superior frontal gyrus & Right & 10 & 3.34 \\
\hline & Middle frontal gyrus & Right & 46 & 3.15 \\
\hline & Cingulate (frontal portion) & Left & 32 & 2.95 \\
\hline & Precuneus & Left & 7 & 3.41 \\
\hline & Fusiform gyrus & Left & 37 & 3.20 \\
\hline & Lingual gyrus & Left & 18,19 & 3.20 \\
\hline & Cuneus & Left & 17,18 & 3.30 \\
\hline \multirow{4}{*}{6 months $<3$ months } & Inferior frontal gyrus, & Left & 47 & 3.60 \\
\hline & Superior temporal gyrus (temporal pole) & Left & 38 & 3.60 \\
\hline & Middle temporal gyrus & Left & 21 & 3.10 \\
\hline & Superior temporal gyrus (posterior) & Right & 22 & 3.38 \\
\hline
\end{tabular}

a. The regions with significant changes in blood flow between 0 (baseline) and 3 months and 3 and 6 months after treatment initiation are listed. $\mathrm{Z}$ scores represent the biggest changes in blood flow across voxels within the region. 


\section{Discussion}

\subsection{Summary of Results}

We found that $\mathrm{rCBF}$ values in the superior temporal gyrus (temporal pole), cingulate (frontal portion), and inferior frontal gyrus were consistently lower in patients with major depression compared to controls throughout the 6-month treatment period (Table 2). In terms of symptoms, at least 6 months of treatment is considered necessary for full recovery from depression [7]. During the 6-month period, $\mathrm{rCBF}$ of the patients consistently increased in the superior and middle frontal gyri (Table 3). At 6 months, patients showed higher rCBF ratios than the controls in the middle temporal gyrus, superior temporal gyrus, fusiform gyrus, middle occipital gyrus, lingual gyrus, and cuneus (Table 2).

Baseline rCBF in our patients was lower than that in controls in a wide range of the frontal regions. While depressive symptoms were significantly improved at 3 months, rCBF ratios in the superior and middle frontal gyri increased. However, $\mathrm{rCBF}$ ratios in the middle and inferior frontal regions remained low. At 6 months, depressive symptoms continued to improve, and rCBF ratios increased in the superior frontal gyrus, middle frontal gyrus, cingulate (frontal portion), precuneus, fusiform gyrus, lingual gyrus, and cuneus. Conversely, rCBF ratios decreased in the inferior frontal gyrus, superior temporal gyrus (temporal pole), middle temporal gyrus, and superior temporal gyrus.

\subsection{Advantages of Our Study}

In the present study, $\mathrm{rCBF}$ increased in the left middle and superior frontal gyri after 3 months, and those on the right side increased more after 6 months. rCBF decreased in the superior temporal gyrus (temporal pole) including the amygdala after 6 months. It remained unchanged in the cingulate gyrus after 3 months but increased after 6 months. Thus, there were temporal differences in brain activity changes represented by rCBF over the course of treatment.

A period of at least 6 months is thought to be required to recover from a major depressive episode [7]. Previous studies on $\mathrm{rCBF}$ in depression compared patients with healthy controls at a single time point or assessed rCBF changes before and after treatment. There have been some observations performed at three or more time points in the course of treatment, but all of these were case reports. The present study investigated changes in symptoms and $\mathrm{rCBF}$ at baseline and 3 and 6 months after treatment, which allows us to provide more accurate information regarding $\mathrm{rCBF}$ changes following treatment.

\section{3. rCBF in Depression}

There have many previous studies on $\mathrm{rCBF}$ using SPECT in patients with major depression [1]-[6]. To our knowledge, three have compared patients with healthy controls [1] [3] [18]. Oda et al. used ${ }^{99 \mathrm{~m}} \mathrm{Tc}-\mathrm{ECD}$ and reported lower $\mathrm{rCBF}$ in the frontal and temporal lobes and anterior cingulate gyrus in patients [3]. In line with this, Bonne and colleagues used ${ }^{99 \mathrm{~m}} \mathrm{Tc}-\mathrm{HMPAO}$ and reported 
that $\mathrm{rCBF}$ was lower in the right parietal, occipital, and left temporal lobes in patients compared with controls [1]. Interestingly, Pagani et al. also used ${ }^{99 \mathrm{~m}} \mathrm{Tc}$ HMPAO and found relatively higher $\mathrm{rCBF}$ in the right temporo-parietal cortex, bilateral prefrontal and frontal cortices, and anterior temporal cortex [18].

Both Arthur et al. and Mayberg et al. conducted comparative studies with healthy controls and patients with major depression using positron emission tomography (PET) [10] [19]. Mayberg et al. reported higher CBF in the subgenual cingulate and lower $\mathrm{CBF}$ in the prefrontal, premotor, dorsal anterior cingulate, and anterior insular regions in patients with major depression compared with controls using ${ }^{15} \mathrm{O}$ as a tracer [19]. Arthur and colleagues used fludeoxyglucose (FDG) and found higher glucose metabolism in the prefrontal cortex, caudate, and thalamus and lower glucose metabolism in the temporal lobe of patients compared to controls [4]. Drevets observed high blood flow in the amygdala before treatment [5].

In summary, the previous reports are inconsistent regarding some areas including the prefrontal, frontal, temporal, and parietal cortices. Among them, the temporal cortex is consistently reported to show reduced $\mathrm{rCBF}$ in depression, with three studies reporting lower rCBF in patients [1] [3] [4], but discrepant results were reported by Pagani et al. In contrast, the anterior cingulate is consistently reported to have lower rCBF in patients than in controls [3] [19]. The premotor, anterior insula, and occipital cortex are reported to show lower $\mathrm{rCBF}$, while the subgenual cingulate, caudate, thalamus, and amygdala display higher rCBF in depression than controls [4] [5] [19].

\section{4. rCBF Changes Following Treatment for Major Depression}

Davies at al. conducted a SPECT study with ${ }^{99 \mathrm{~m}} \mathrm{Tc}$-HMPAO that assessed the effect of treatment on $\mathrm{rCBF}$ in patients with major depression [2]. They reported increased $\mathrm{rCBF}$ in the bilateral thalamus and decreased $\mathrm{rCBF}$ in the right posterior temporal, left occipital lobe, and cerebellum following venlafaxine treatment. Three groups show changes in PET tracer levels following treatment [4] [19] [20]. Arthur et al. reported normalization of FDG in the prefrontal cortex, caudate, and thalamus to levels comparable with healthy control group following various treatments [4], and Mayberg et al [19]. reported normalization of rCBF to levels comparable with controls in the subgenual cingulate and prefrontal cortex of treated patients. Mayberg [20] also reported that treatment elevated blood flow in the anterior cingulate gyrus. Kennedy et al. investigated brain metabolism using PET before and after 16-week treatment for major depression [21]. They found reduced brain metabolism in the ventrolateral prefrontal cortex.

At the start of treatment, the left and right superior temporal gyrus (temporal pole) had low rCBF in the patient group (Table 2). The amygdala is thought to be included in the temporal pole. A further decrease in $\mathrm{rCBF}$ of the left superior temporal gyrus (temporal pole) was seen after treatment (Table 3). Our observation of treatment-related decrease in $\mathrm{rCBF}$ in the left temporal pole including 
the amygdala has not been reported in previous SPECT studies of patients with depression. However, fMRI [22] and PET [20] [23] studies have described reduced activity in the left amygdala of patients with depression after treatment. This suggests a relationship between strong negative feelings and high amygdalar activity. Consequently, the treatment-related decrease in rCBF of the left amygdala is an intriguing result and may hint at the amygdala's pathophysiologic role in depression.

In the present study, the different brain areas of depressed patients demonstrated two different patterns of blood flow change during recovery from depression. In the superior and middle frontal gyri and cingulate, blood flow increased, while it decreased in the superior temporal gyrus and temporal pole including the amygdala (Table 3). Interestingly, these areas are all associated with emotion. This suggests that different brain areas related to emotions would are differentially involved in the pathogenesis of depression.

Previous brain imaging studies on depression can be summarized in that there were two common findings after successful treatment: 1) increased metabolism in the DLPFC and 2) decreased metabolism in the amygdala [5] [19] [21]. However, a major problem with these previous studies is that none found both increased and decreased blood flow changes in the same subject group. The present study is unique in that we found opposite changes in blood flow in a single group of patients during the course of treatment. To our knowledge, the findings of 1) a relatively early increase in blood flow in the superior and middle frontal gyri corresponding to the DLPFC and 2) a relatively late decrease in the amygdala have not been previously reported.

\subsection{Time Difference of Treatment Response in Different Areas of the Social Cognitive Neural Network}

In the present study, areas in which higher rCBF was observed in patients compared to controls at 3 months included the superior temporal gyrus, inferior temporal gyrus, middle temporal gyrus, middle occipital gyrus, and fusiform gyrus. At 6 months, rCBF increased more in these areas (Figure 2). The fusiform and superior temporal and middle temporal gyri are part of the social cognitive neural network [24] [25]. Therefore, it is speculated that multiple brain regions related with social cognition are differentially activated during recovery from a depressive state. Social cognition is thought to be involved in deficits associated with depression, which are expected to recover with clinical improvement. The recovery process from disordered social cognition is speculated to be gradual because the recovery processes of the neural substrates of social cognition are gradual, as shown in the above-cited results.

\subsection{Possible Effect of Age on rCBF}

The mean ages of the control and patient groups were $31.5 \pm 12.8$ and $46.3 \pm$ 13.2 years, respectively, which were significantly different. Therefore, the effect of age is considered a potential problem. The literature contains several reports 
on the influence of age on CBF flow in healthy adults. Ances et al. [26] reported that the mean elevation rate of $\mathrm{CBF}$ and cerebral metabolic rate of $\mathrm{O}_{2}$ in response to visual stimuli was the same in 10 subjects with a mean age of 28 years and 10 with a mean age of 53 years; thus, age did not seem to affect the response to stimuli [26]. Giovacchini et al. reported no differences in whole gray-matter $\mathrm{CBF}$ among eight people with a mean age of 27 years and seven subjects with a mean age of 65 years after partial volume correction [27]. Similarly, Meltzer et al. [28] reported no difference in cortical CBF between groups aged 19 - 46 and 60 - 76 years after partial volume correction. These reports suggest that there is no significant age-dependent difference in $\mathrm{rCBF}$ in healthy adults.

Nagafusa et al. assessed the effect of age on rCBF in patients with depression using SPECT imaging [29]. They did not find an effect of age on rCBF decreases in the depression group; rather, the reductions were common to all ages, specifically in subjects from 30 to 79 years old. Based on the above-cited studies, we tentatively conclude that the age difference between the two groups in the present study did not critically distort a real difference between depressive patients and healthy control subjects.

Actually, Terada et al [30] compared rCBF in old patients with depression, very old patients with depression, and healthy controls. They found differences in the frontal lobe, thalamus, and entire cerebrum, but the differences in the cerebellum were not statistically significant. If an age difference of $\mathrm{rCBF}$ exists at all, its effect should be cancelled, because $\mathrm{rCBF}$ is expressed was the standardized ratio of cerebrum/cerebellum in the present study.

\subsection{Study Limitations}

The following points should be considered when interpreting the present results. The number of patients was limited, and the control group was significantly more educated than the patient group. Frontal lobe function is reported to have a general correlation with years of education [31], but there are also reports of negative correlation between frontal lobe activity and years of education in some young people and a positive correlation in elderly subjects [32]. Thus, we cannot rule out an effect of education on frontal lobe function between the patient and control groups.

\section{Conclusions}

Our results showed increased blood flow in the superior and middle frontal gyri and middle temporal gyrus and decreased blood flow in the superior temporal gyrus at 3 months, as well as decreased blood flow in the temporal pole including the amygdala at 6 months after initiation of treatment for major depressive disorder. This is the first study to report these three findings in a single group of subjects.

Our results confirm that $\mathrm{rCBF}$ is lower in the frontal lobe and temporal pole of patients with major depression as compared to the controls prior to treatment. After treatment initiation, $\mathrm{rCBF}$ of the posterior temporal lobe in patients 
increased to a higher level than that observed in control, while rCBF of the temporal pole was further decreased in patients. The patients' rCBF changes are thought to reflect functional improvement in cognition involving the frontal lobe and social cognition mediated by the temporal lobe. The decrease in patients' temporal lobe rCBF may be due to less anxiety or discomfort in the course of treatment; thus, $\mathrm{rCBF}$ of the temporal pole including the amygdala, which plays a major role in controlling emotion, is lowered. By taking serial rCBF measurements at fixed times following the initiation of treatment for major depressive disorder, we provide the first evidence that there are temporal and regional differences in $\mathrm{rCBF}$ recovery in major depression. Thus, the extent of recovery from depression can be objectively monitored, and it may be possible to obtain objective data for antidepressant dose reduction and discontinuation.

\section{References}

[1] Bonne, O., Louzoun, Y., Aharon, I., Krausz, Y., Karger, H., Lerer, B., Bocher, M., Freedman, N. and Chisin R. (2003) Cerebral Blood Flow in Depressed Patients: A Methodological Comparison of Statistical Parametric Mapping and Region of Interest Analyses. Psychiatry Research, 122, 49-57. https://doi.org/10.1016/S0925-4927(02)00103-8

[2] Davies, J., Lloyd, K.R., Jones, I.K., Barnes, A. and Pilowsky, L.S. (2003) Changes in Regional Cerebral Blood Flow with Venlafaxine in the Treatment of Major Depression. The American Journal of Psychiatry, 160, 374-376. https://doi.org/10.1176/appi.ajp.160.2.374

[3] Oda, K., Okubo, Y., Ishida, R., Murata, Y., Ohta, K., Matsuda, T., Matsushima, E., Ichimiya, T., Suhara, T., Shibuya, H. and Nishikawa, T. (2003) Regional Cerebral Blood Flow in Depressed Patients with White Matter Magnetic Resonance Hyperintensity. Biological Psychiatry, 53, 150-156.

https://doi.org/10.1016/S0006-3223(02)01548-2

[4] Brody, A.L., Saxena, S., Stoessel, P., Gillies, L.A., Fairbanks, L.A., Alborzian, S., Phelps, M.E., Huang, S.C., Wu, H.M., Ho, M.L., Ho, M.K., Au, S.C., Maidment, K. and Baxter Jr., L.R. (2001) Regional Brain Metabolic Changes in Patients with Major Depression Treated with Either Paroxetine or Interpersonal Therapy. Archives of General Psychiatry, 58, 631-640. https://doi.org/10.1001/archpsyc.58.7.631

[5] Drevets, W.C. (2000) Functional Anatomical Abnormalities in Limbic and Prefrontal Cortical Structures in Major Depression. Progress in Brain Research, 126, 412 431. https://doi.org/10.1001/archpsyc.58.7.631

[6] Brody, A.L., Barsom, M.W., Bota, R.G. and Saxena, S. (2001) Prefrontal-Subcortical and Limbic Circuit Mediation of Major Depressive Disorder. Seminars Clinical Neuropsychiatry, 6, 102-112.

[7] Brown, C., Schulberg, H.C., Madonia, M.J., Shear, M.K. and Houck, P.R. (1996) Treatment Outcomes for Primary Care Patients with Major Depression and Lifetime Anxiety Disorders. The American Journal of Psychiatry, 153, 1293-1300. https://doi.org/10.1176/ajp.153.10.1293

[8] American Psychiatric Association (1994) Diagnostic and Statistical Manual of Mental Disorders. 4th Edition, APA, Washington DC.

[9] Hamilton, M. (1960) A Rating Scale for Depression. Journal of Neurology Neurosurgery and Psychiatry, 23, 56-62. https://doi.org/10.1136/jnnp.23.1.56

[10] Buchpiguel, C., Alavi, A., Crawford, D., Freeman, E. and Newberg A. (2000) 
Changes in Cerebral Blood Flow Associated with Premenstrual Syndrome: A Preliminary Study. Journal of Psychosomatic Obstetrics \& Gynecology, 21, 157-165. https://doi.org/10.3109/01674820009075623

[11] Erkwoh, R., Sabri, O., Willmes, K., Steinmeyer, E.M., Bull, U. and Sass, H. (1999) Aspects of Cerebral Connectivity in Schizophrenia. A Comparative CBF Study on Treated Schizophrenics before and after Medication. Fortschritte der NeurologiePsychiatrie, 67, 318-326. https://doi.org/10.1055/s-2007-994982

[12] Galynker, I.I., Cai, J., Ongseng, F., Finestone, H., Dutta, E. and Serseni D. (1998) Hypofrontality and Negative Symptoms in Major Depressive Disorder. The Journal of Nuclear Medicine, 39, 608-612.

[13] Sabri, O., Erkwoh, R., Schreckenberger, M., Cremerius, U., Owega, A., Dickmann, C., Schulz, G., Zimny, M., Sass, H. and Buell, U. (1997) Altered Relationships between rCBF in Different Brain Regions of Never-Treated Schizophrenics. Nuklearmedizin, 36, 194-201.

[14] Bonne, O., Krausz, Y., Shapira, B., Bocher, M., Karger, H., Gorfine, M., Chisin, R. and Lerer, B. (1996) Increased Cerebral Blood Flow in Depressed Patients Responding to Electroconvulsive Therapy. The Journal of Nuclear Medicine, 37, 1075 1080.

[15] Kanaya, T. and Yonekawa, M. (1990) Regional Cerebral Blood Flow in Depression. The Japanese Journal of Psychiatry and Neurology, 44, 571-576. https://doi.org/10.1111/j.1440-1819.1990.tb01631.x

[16] Jack, L.L., Marty, G.W., Lawrence, M.P., Mario, L., Catarina, S.F., Lacy, R., Peter, V.K., Dan, N., Shawn, A.M. and Peter, T.F. (2000) Automated Talairach Atlas Labels for Functional Brain Mapping. Human Brain Mapping, 10, 120-131. https://doi.org/10.1002/1097-0193(200007)10:3<120::AID-HBM30>3.0.CO;2-8

[17] Inagaki, A., Inada, T. and Fujii, Y. (1999) Dose Equivalents of Psychotropic Drugs. Seiwa Press, Tokyo. (In Japanese)

[18] Pagani, M., Gardner, A., Salmaso, D., Sánchez, C.A., Jonsson, C., Jacobsson, H., Lindberg, G., Wägner, A., Hällström, T. and Larsson, S.A. (2004) Principal Component and Volume of Interest Analyses in Depressed Patients Imaged by 99mTcHMPAO SPET: A Methodological Comparison. European Journal of Nuclear Medicine and Molecular Imaging, 31, 995-1004. https://doi.org/10.1007/s00259-004-1457-5

[19] Mayberg, H.S., Lozano, A.M., Voon, V., McNeely, H.E., Seminowicz, D., Hamani, C., Schwalb, J.M. and Kennedy, S.H. (2005) Deep Brain Stimulation for Treatment-Resistant Depression. Neuron, 45, 651-660. https://doi.org/10.1016/j.neuron.2005.02.014

[20] Mayberg, H.S. (2003) Positron Emission Tomography Imaging in Depression: A Neural Systems Perspective. Neuroimaging Clinics of North America, 13, 805-815. https://doi.org/10.1016/S1052-5149(03)00104-7

[21] Kennedy, S.H., Konarski, J.Z., Segal, Z.V., Lau, M.A., Bieling, P.J., McIntyre, R.S. and Mayberg, H.S. (2007) Differences in Brain Glucose Metabolism between Responders to CBT and Venlafaxine in a 16-Week Randomized Controlled Trial. American Journal of Psychiatry, 164, 778-788. https://doi.org/10.1176/ajp.2007.164.5.778

[22] Peluso, M.A., Glahn, D.C., Matsuo, K., Monkul, E.S., Najt, P., Zamarripa, F., Li, J., Lancaster, J.L., Fox, P.T., Gao, J.H. and Soares, J.C. (2009) Amygdala Hyperactivation in Untreated Depressed Individuals. Psychiatry Research, 173, 158-161. https://doi.org/10.1016/j.pscychresns.2009.03.006

[23] Drevets, W.C., Videen, T.Q., MacLeod, A.K., Haller, J.W. and Raichle, M.E. (1992) 
PET Images of Blood Flow Changes during Anxiety: Correction. Science, 256, 1696. https://doi.org/10.1126/science.256.5064.1696

[24] Pelphrey, K., Adolphs, R. and Morris, J.P. (2004) Neuroanatomical Substrates of Social Cognition Dysfunction in Autism. Mental Retardation and Developmental Disabilities Research Reviews, 10, 259-271. https://doi.org/10.1002/mrdd.20040

[25] Chao, Z., Nagasaka, Y. and Fujii, N. (2015) Cortical Network Architecture for Context Processing in Primate Brain. eLIFE, 4, e06121. https://doi.org/10.7554/elife.06121

[26] Ances, B.M., Liang, C.L., Leontiev, O., Perthen, J.E., Fleisher, A.S., Lansing, A.E. and Buxton, R.B. (2009) Effects of Aging on Cerebral Blood Flow, Oxygen Metabolism, and Blood Oxygenation Level Dependent Responses to Visual Stimulation. Human Brain Mapping, 30, 1120-1132. https://doi.org/10.1002/hbm.20574

[27] Giovacchini, G., Lerner, A., Toczek, M.T., Fraser, C., Ma, K., DeMar, J.C., Herscovitch, P., Eckelman, W.C., Rapoport, S.I. and Carson, R.E. (2004) Brain Incorporation of ${ }^{11} \mathrm{C}$-Arachidonic Acid, Blood Volume, and Blood Flow in Healthy Aging: A Study with Partial-Volume Correction. The Journal of Nuclear Medicine, 45, 1471 1479.

[28] Meltzer, C.C., Cantwell, M.N., Greer, P.J., Ben-Eliezer, D., Smith, G., Frank, G., Kaye, W.H., Houck, P.R. and Price, J.C. (2000) Does Cerebral Blood Flow Decline in Healthy Aging? A PET Study with Partial-Volume Correction. The Journal of Nuclear Medicine, 41, 1842-1848.

[29] Nagafusa, Y., Okamoto, N., Sakamoto, K., Yamashita, F., Kawaguchi, A., Higuchi, T. and Matsuda, H. (2012) Assessment of Cerebral Blood Flow Findings Using 99mTc-ECD Single-Photon Emission Computed Tomography in Patients Diagnosed with Major Depressive Disorder. Journal of Affective Disorders, 140, 296 299. https://doi.org/10.1016/j.jad.2012.03.026

[30] Terada, T., Uno, M., Motohashi, N., Matsuda, H., Nakano, M., Takano, H., Morooka, T. and Asada, T, (1997) Noninvasive Measurements of Cerebral Blood Flow Using 99mTc-ECD SPECT in Elderly Depressed Patients. Clinical Psychiatry, 39, 735-742. (In Japanese)

[31] Abe, M., Suzuki, K., Okada, K., Miura, R., Fujii, T., Etsurou, M. and Yamadori, A. (2004) Normative Data on Tests for Frontal Lobe Functions: Trail Making Test, Verbal Fluency, Wisconsin Card Sorting Test (Keio Version). No To Shinkei, 57, 567-574. (In Japanese)

[32] Springer, M.V., McIntosh, A.R., Winocur, G. and Grady, C.L. (2005) The Relation between Brain Activity during Memory Tasks and Years of Education in Young and Older Adults. Neuropsychology, 19, 181-192.

https://doi.org/10.1037/0894-4105.19.2.181 
Submit or recommend next manuscript to SCIRP and we will provide best service for you:

Accepting pre-submission inquiries through Email, Facebook, LinkedIn, Twitter, etc. A wide selection of journals (inclusive of 9 subjects, more than 200 journals)

Providing 24-hour high-quality service

User-friendly online submission system

Fair and swift peer-review system

Efficient typesetting and proofreading procedure

Display of the result of downloads and visits, as well as the number of cited articles Maximum dissemination of your research work

Submit your manuscript at: http://papersubmission.scirp.org/

Or contact ojpsych@scirp.org 